\title{
EQUATING CATEGORIALLY NAMES AND QUANTIFIERS WITHIN FIRST-ORDER LOGIC
}

Grammarians traditionally consider quantifier expressions such as something, everything, somebody, everybody, some dog, every dog together with names (singular name expression) as belonging to the noun phrases category. The reason is simple: both quantifier expressions and names can be used as subjects in sentences. Logicians on the other hand, usually treat quantifier expression (in short: quantifiers) as operators, i.e. as expressions of a different category from the name category. The source of this distinction seems clear: names are categorametic, whereas operators are syncategorsmetic expressions. However, when we are trying to explain the opposition categorameticsyncategorametic we usually give names and quantifiers as paradigmatic examples respectively.

Despite this tradition, one can study logical differences between names and quantifiers on a deductive level while letting them to be expressions of the same category (e.g. the generalized quantifier category). It means that names and quantifiers can be accounted as sharing some syntactic properties while differing in others. As we will see, the advantage of this approach is that it may lead to a version of first-order logic which can accommodate a vast class of expressions included between names and quantifiers.

In order to equate categorically names and quantifiers feasible we must first unify the grammar of these expressions. It is enough to assume that names occupy the same position as quantifiers in the first-order language. 
Thus instead of $P a$ we will have from now on $a x P x$; generally for every formula $A(x)$ we will also have the subject-predicate formula $a x A(x)$. This notation may seem to be strange but basically it is equivalent to a more familiar, but a little bit more complicated $\lambda$-notation: $[\lambda x A(x)] a$.

For convenience, we will use the word 'names' whether talking about singular names or individual constants. Quantifiers and names will be called collectively terms, i.e., terms are either quantifiers or names. Assume that $t$, $s$ represent terms. Let us consider first the following group of properties:

$\mathrm{P} 1 \quad t x \neg A \supset \neg t x A$

$\mathrm{P} 2 \quad \neg t x A \supset t x \neg A$

P3 $t x(A \wedge B) \supset t x A \wedge t x B$

$\mathrm{P} 4 \quad t x A \wedge t x B \supset t x(A \wedge B)$

P5 txA $A \vee x B \supset t x(A \vee B)$

P6 $t x(A \vee B) \supset t x A \vee t x B$

P7 $\quad t x(A \supset B) \supset(t x A \supset t x B)$

P8 txsyA $\supset \operatorname{sytxA}$

P9 $\quad \operatorname{txty} A \supset t x A(x \mid y)$

$\mathrm{P} 10 \quad t x A(x \mid y) \supset t x t y A$

$(A(y \mid x)$ is the formula which results from freely substituting every occurrence of $x$ by $y$ in $A$.)

This is not a complete list of properties (see below) and these properties are not independent from each other assuming classical dependences between connectives, in particular, P5-P7 easily follow from P1-P4. Clearly, names fulfill all principles listed above - it means that names are scope independent (in other words, this is tantamount to the fact that the principle of eliminability of $\lambda$-expressions holds for names: $[\lambda x A(x)] a \equiv A(a \mid x){ }^{1}{ }^{1}$ The universal quantifiers fulfills: P1, P3, P4, P5, P7, P8 (when assigned to $s$ ), and P9. The existential quantifiers fulfills: P2, P3, P5, P6, P8 (when assigned to $t$ ), and $\mathrm{P} 10$. We notice that $\mathrm{P} 3$, and $\mathrm{P} 5$ are fulfilled by all expressions under consideration. On the other hand, it is also worth noticing that indi-

\footnotetext{
${ }^{1}$ This is the reason why we adopt in classical logic much simpler grammar for individual constants than for quantifiers.
} 
vidual constants have all properties among P1-P10 that are possessed either by the universal or by existential quantifier.

Another group of formulas expresses the behavior of all terms in freebound variable relevant contexts.

P11 $t x(A \supset B) \equiv(A \supset t x B)$, where $x$ is not free in $A$;

P12 $t x A \equiv A$, where $x$ is not free in $A$;

P13 txA $\equiv t y A^{*}$, where ${ }^{*}$ differs from $A$ in that $x$ is free in $A$ just in those places where $y$ is free in $A^{*}$.

(Remarkably, there are no properties of this kind which are distinctive for quantifiers and for individual constants).

Still another group of properties can be taken into account; these can be called logical or deductive properties. In particular, they express logical relations between universal quantifier and all terms. The choice of these properties, as properties given before, is not systematic (the list is neither independent nor exhaustive).

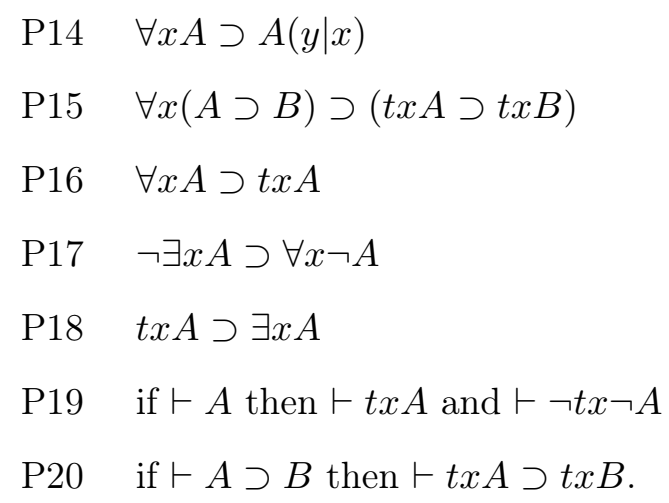

Selecting some of properties P1-P20 given above, possibly adding some new, one can develop a system of first-order logic in which names and quantifiers are expressions of the same category. Theorems of this system are supposed to express all properties specific for names, and no other properties. There are many ways developing an axiomatic system for such logic. In particular, we propose the following system, call it $L$-system.

L1 Classical truth-functional tautologies.

L2 $\forall x(A \supset B) \supset(t x A \supset t x B)$

L3 $A \supset \forall x A$, provided $x$ is not free in $A$ 
L4 $\forall x A \supset A(x \mid y)$

L5 txA $\supset \operatorname{ty} A(y \mid x)$, provided $y$ is not free in $A$

L6 $\neg \exists x A \supset \forall x \neg A$

L7 $\quad \exists x A(x \mid y) \supset($ txty $A \supset t x A(x \mid y))$

L8 $\quad \exists x \neg A(x \mid y) \supset(t x A(x \mid y) \supset t x t y A)$

MP $\quad$ if $\vdash A \supset B$ then $\vdash B$

MG if $\vdash A$ then $\vdash t x A$ and $\vdash \neg t x \neg A .^{2}$

Notice that L7, L8 are quite technical and, as they stand, have not appeared earlier (compare them with P9, P10 which do not hold for all terms).

We see that some axioms of $L$-system resemble, if not directly coincide with, axioms and theorems of classical logic. One can easily check that if we put for $t$ either the universal or the existential quantifier we get a classical law or a classical rule of deduction. All formulas that we mentioned earlier as common properties of names and quantifiers are theorems of $L$ logic. Moreover, the following formulas are theses of $L$-logic:

$$
\begin{array}{ll}
\text { L9 } & (\neg t x C \supset t x \neg C) \vee(t x \neg C \supset t x \neg C) \\
\text { L10 } & (t x A \wedge \neg \forall x A) \wedge(\neg t x B \wedge \exists x B) \supset(\neg t x C \equiv t x \neg C) \\
\text { L11 } & (t x A \wedge \neg \forall x A) \wedge(\neg t x B \wedge \exists x B) \supset((t x C \wedge t x D) \equiv t x(C \wedge D))^{3} \\
\text { L12 } & (t x A \wedge \neg \forall x A) \wedge(\neg t x B \wedge \exists x B) \supset(\operatorname{sxty} C \equiv t y s x C) \\
\text { L13 } & (t x A \wedge \neg \forall x A) \wedge(\neg t x B \wedge \exists x B) \supset(t x t y C \equiv t x C(x \mid y))
\end{array}
$$

\footnotetext{
${ }^{2}$ This rule of inference can be replaced by the ordinary rule of generalization: if $\vdash A$ then $\vdash \forall x A$, but then we must also add an additional axiom $\mathrm{P} 12$.

${ }^{3}$ Actually, L9 and L10 follow from the following theorems:

$$
\begin{aligned}
(t x A & \wedge \forall x A) \\
(\neg t x B \wedge(\neg t x C \supset \neg C) & \supset(t x \neg C \supset \neg t x C) \\
(\neg t x B & \wedge \exists x B) \\
t x(C \wedge D) & \supset(t x C \wedge t x D) \supset t x(C \wedge D) \\
& (t x C \wedge t x D)
\end{aligned}
$$
}


Roughly speaking, theses L10-L13 say that if a term coincides neither with the universal nor existential quantifier then its scope does not matter and this is what we exactly expect from individual constants.

The semantic for $L$-logic, $L$-semantic, is quite similar to that for classical first-order language following standards of Tarski's semantics. By a model, we mean a pair $m=[D, I]$ where $D$ is a non-empty set called the domain of interpretation, $I$ is a function defined on terms and predicates called the interpretation:

(a) For an individual constant $a, I(a)=\{\{d\}\}$, where $d \in D, I(\forall)=\{D\}$, $I(\exists)=\wp(D)-\{\emptyset\}$ (here $\wp(D)$ is the power set of $D$ );

(b) $I(P) \subseteq D^{\mathrm{n}}$, for $n$-argument predicate symbol $P$

An assignment in $D$ is a function $V$ which assigns to every variable an element of $D$. given $V$, by $V[d / x]$ we mean the function which is just like $V$, except possibly $V[d / x] x=d$.

Truth conditions for atomic formulas, for negation and implication, are the same as in classical semantics.

$$
\begin{aligned}
& \text { (1) }\left\|P y_{1} \ldots y_{n}\right\|_{V}^{m}= \begin{cases}1 & \text { iff }\left[V\left(y_{1}\right), \ldots, V\left(y_{n}\right)\right] \in I(P), \\
0 & \text { otherwise; }\end{cases} \\
&\|x=y\|_{V}^{m}= \begin{cases}1 & \text { iff } V(x)=V(y), \\
0 & \text { otherwise. }\end{cases} \\
& \text { (2) } \quad\|\neg A\|_{V}^{m}=1-\|A\|_{V}^{m} \text { (3) } \quad\|A \supset B\|_{V}^{m}=\max \left[1-\|A\|_{V}^{m},\|B\|_{V}^{m}\right] \\
& \text { (4) } \quad\|t x A\|_{V}^{m}=1 \quad \text { iff } \quad \bigvee_{X \in I(t)} X \subset I_{V}(x A), \\
& \quad \text { where } I_{V}(x A)=d \in D:\|A\|_{V[d / x]}^{m}=1
\end{aligned}
$$

In particular, the formula $t x P x$ is true in $m$ iff there exists $X \in I(t)$ such that $X \subseteq I(P)$. Notice first that quantifiers receive in $L$-semantics independent interpretations what could be understood as categorematic treatment of these expressions. And their interpretations are of the same kind as interpretations of individual constants, i.e., both are subsets of $\wp(D) .{ }^{4}$ Thus we may say

\footnotetext{
${ }^{4}$ This semantical idea is not quite new. It can be found in Richard Montague's works, cf. Montague [1974]. See also: Dowty [1981]. The idea of treating quantifier expressions as categorematic, i.e., as representing a kind of entities called 'quantifier objects' goes back to medieval logic. However, Frege's approach to quantifiers as second-order concepts may also be associated with categorematic reading these expressions.
} 
that names and quantifiers belong to the same semantic category according to $L$-semantics. Notice also that the condition (4) retains the usual meaning of the universal and existential quantifiers. A formula is valid iff it is true in every model with respect to any assignment.

Undoubtly, $L$-logic is, in some reasonable sense, equivalent to the classical logic. ${ }^{5}$ Accordingly we can conclude that quantifiers and constants can be treated as expressions of the same syntactic category in a broadly conceived classical logic. And individual constants are distinguished on the deductive level. But is this all we can obtain from the uniform categorical treatment of these two groups of expressions? One can consider $L$-logic as excessively complicated. This complication may not seem as overweighing its richer logical contents.

It turns out, however, that the $L$-logic, even if not considered as particularly interesting, can reveal a wider logical perspective. Let us have a look again at the deductive system and semantics of $L$-logic. Axioms M7 and M8 are strikingly unnatural and their content is unclear. Why don't drop these axioms then? What will result we call $M$-logic:

M1 Classical truth functional tautologies

M2 $\forall x(A \supset B) \supset(t x A \supset t x B)$

M3 $A \supset \forall x A$, provided $x$ is not free in $A$

M4 $\forall x A \supset A(y \mid x)$

M5 txA $\supset \operatorname{ty} A(y \mid x)$, provided $y$ is not free in $A$

M6 $\neg \exists x A \supset \forall x \neg A$

MP $\quad$ if $\vdash A \supset B$ and $\vdash A$ then $\vdash B$

MG if $\vdash A$ then $\vdash t x A$ and $\vdash \neg t x \neg A$.

\footnotetext{
${ }^{5}$ Let us define the translation $T$ of formulas of $L$-language onto formulas of classical language:

(a) $T(A)=A$, if $A$ is an atomic formula;

(b) $T(\neg A)=\neg T(A)$;

(c) $T(A \wedge B)=T(A) \wedge T(B)$;

(d) $T(a x A)=T(A(a \mid x))$;

(e) $T(Q x A)=Q x T(A)$, for a quantifier $Q$.

Then for every formula $A$ of $L$-language: $A$ is a thesis of $L$-system iff $T(A)$ is classical thesis. Obviously, a classical formula can be the translation of many different but equivalent formulas of $L$-language.
} 


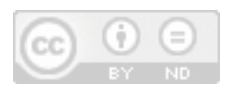

EQUATING CATEGORIALly NAMES . .

Perhaps the effect of this impoverishment of $L$-logic is not directly seen. But it will become much more clear when we equip $M$-logic with a semantics. Let us remember that in $L$-semantics interpretations of constants and quantifiers were of the same kind in the sense that they were subsets of $\wp(D)$. But obviously there are a lot of other subsets of $\wp(D)$ which are unexploited in $L$-semantics, i.e., cannot be interpretations of individual constants or quantifiers. So let us assume that the first condition of interpretations will look now in the following way ( $L$-language becomes $M$-language):

$\left(\mathrm{a}^{*}\right) \quad I(t) \subseteq \wp(D), I(t) \neq \emptyset$ and $I(t) \neq\{\emptyset\}$; in particular $I(\forall)=\{D\}$, $I(\exists)=\wp(D)-\{\emptyset\}$.

Thus $\left(\mathrm{a}^{*}\right)$ extends the category of terms. It means that almost all subsets of $\wp(D)$ can be interpretations of terms in $M$-semantics. ${ }^{6}$ So what kind of terms are they supposed to be? And what do they represent or stand for? Without any danger of getting involved in ontological commitments we may assume that interpretations of terms represent some sort of entities. This makes easier further discussions.

Consider some examples. Suppose that $t$ is a term such that $I(t)=$ set of Poles, set of Popes\}. Then it turns out that $t$ (or, more precisely, the entity represented by $t$ ) possesses, in the sense determined by the truth condition (4), the following properties: being a Pole, being a Pope, being a human, et. but not properties of being tall, being nervous, etc. So $t$ may be conceived here as the description the Polish Pope. If $I(t)=$ s set of idlers, set of students $\}$ then $t$ may be treated as a generic term the lazy student and the object correlated with it, according to $M$-semantics, possesses the properties of being lazy, being student, being human, etc. At the same time, it does not possess many other properties, like being a cat, being a girl, being a boy, etc. Similarly, \{set of circles, set of squares\} can be identified with the round square which according to the semantics is square, is round, is geometrical figure but it is neither green nor non-green. Consider now example of a different kind. The set $\{X \subset D:|X| \geqslant 5\}$ can be interpreted as at least five things; the set $\{X \subset D:|X| \geqslant|D-X|\}$ as most things.

Thus $M$-logic can be understood in various ways depending on how we understand terms. First, we may provide definite description which differentiate meanings or senses of descriptions. We see that the cardinal born in Wadowice interpreted as the set of cardinals, the set of people born in Wadowice\} according to $M$-semantics is distinct then the Polish Pope, i.e., the

${ }^{6}$ We exclude $\emptyset$ and $\{\emptyset\}$ mainly for some technical reasons. 
cardinal born in Wadowice possesses different properties: being a cardinal, being born in Wadowice, but neither being a Pole, nor being a Pope). ${ }^{7}$ At the same time we have semantic and syntactic means to render the fact that the Polish Pope and the cardinal borne in Wadowice is the same person: notice that the intersection of elements of both interpretations is the same: $\{$ Karol Wojtyła $\}$. This means that both descriptions refer to Karol Wojtyła and, what is important, the identity of reference is expressible in $M$-language. ${ }^{8}$

We may consider $M$-logic as a logic of non-existent objects and this is perhaps the most important interpretation of it. The problem of non-existent objects is certainly one of the most recalcitrant problems of contemporary analytic philosophy, especially challenging for logic. So in particular, the theory of description, free logics, and more recently Meinongian logics are devised to cope with this problem. Unlike the classical theory of description which makes trivial any predication concerning non-existent object, in $M$-logic some predications are true, some are false, in accordance with our intuitions (see the example above). At the same time, $M$-logic fulfills the classical definition of free logics. ${ }^{9}$ But perhaps most of all, $M$-logic is akin in its ontological spirit to Meinong's theory of objects and can be used as a basic logic for developing so-called Meinongian logic. Roughly speaking, according to Meinong every class of properties constitutes an object and we see that in $M$-semantics sets of extensionally represented properties, i.e.,

\footnotetext{
${ }^{7}$ One can say that the difference in meaning of the Polish Pope and the cardinal born in Wadowice can be satisfactorily rendered on the ground of possible worlds semantics because in some worlds these descriptions may denote different objects. Consider, however, another example: the number greater than ten and smaller than twelve and the prime number consisting of two identical digits assuming that natural numbers are only members of the domain. These two descriptions refer in all possible worlds to the number eleven and thus they are indistinguishable with respect to their meanings (intensions) on the ground of possible world semantics. On the other hand, as represented in $M$-semantics by: the set of numbers greater than ten, the set of numbers smaller than twelve\} and the set of prime numbers, the set of numbers consisting of two identical digits $\}$ the objects of these descriptions possess different properties, i.e. those which are mentioned in the descriptions.

${ }^{8}$ In $M$-language the identity of reference of two terms $s$ and $t$ is expressed by a quite complicated formula: $\exists x(s y(x=y) \wedge t y(x=y)) \wedge s x \neg s y(x \neq y) \wedge t x \neg t y(x \neq y)$.

${ }^{9}$ Ernano Bencivenga defines the free logic in the following way:
}

A free logic is a formal system of quantification theory, with or without identity, which allows for some singular terms in some circumstances to be thought of as denoting no existing object, and in which quantifiers are invariably thought of as having existential import. Bencivenga [1986]

Also Karel Lambert, who is recognized as the founder of free logics, agreed that $M$-logic can be classified as a free logic; private conversation. 
represented as sets of individuals, are interpretations of terms. ${ }^{10}$ Our examples also show that terms can be interpreted as generalized quantifiers. ${ }^{11}$ Of course, the logic does not accommodate all generalized quantifiers in the most straightforward manner.

Thus one can see that $M$-logic allows for various kinds of expressions to be subjects of sentences. Accordingly, if we agree on categorematic character of these expressions - such a character is expressed directly by $M$-semantics anyway - then we can say that the logic allows for a various kinds of entities including a broad category of non-existent one. But does that mean that $M$ logic is ontologically committed to the existence of these objects? Hardly. If we accept Quinean criterion of ontological commitment: to be is to be the value of bound variable, then $M$-logic is committed exactly to the same entities as classical first-order logic, i.e., to existing individuals only. So, it is not surprising $M$-logic fulfills the widely accepted Bencivenga's definition of free logics.

Let us turn back to skipped axioms L7, L8. As we noticed, their role in $L$-logic was to exclude all terms except names and (classical) quantifiers. So one can wonder whether it is possible to distinguish individual constants from other terms on the ground of $M$-logic. The answer is that it is, but only when $M$-logic is equipped with identity introduced in the classical way. ${ }^{12} \mathrm{Im}$ portantly, in the extended $M$-logic the formula $\operatorname{txty}(x=y) \wedge(\neg \operatorname{txty}(x \neq y)$ expresses the fact that $t$ is a proper name and, what follows, when we replace the predecessor in $\mathrm{P} 10-\mathrm{P} 13$ by this formula then the resulting formulas will be also theses. It is worth emphasizing that identity also enable us to distinguish among terms still another categories of expressions.

\footnotetext{
10 See: Paśniczek [1998].

11 The notation of generalized quantifiers was defined for the first time by Andrzej Mostowski but it was treated for a long time as a non-essential generalization of the notion of classical quantifier. Nowadays studies of generalized quantifiers are very extensive. On the one hand, the notion of generalized quantifiers turns out to be of particular importance for metalogic. On the other hand, this notion is now frequently applied by linguists in analyses of determiners and noun phrases. Cf. Mostowski [1957], Barwise, Cooper [1981], Chierchia, McConnel-Ginet [1990], Sher [1991], Westerstahl [1989].

12 I.e. adjoin to $M$-system axioms:

Mi $x=y$

Mii $x=y \supset(A \supset A(y \| x))$
}

$(A(y \| x)$ is a formula which results from freely substituting every or only some occurrences of $x$ by $y$ ), and the following condition:

$$
\|x=y\|_{V}^{m}=1 \quad \text { iff } \quad V(x)=V(y),\|x=y\|_{V}^{m}=0 \text { otherwise. }
$$




\section{References}

[1] Barwise, Jon, and Cooper, Richard [1981], "Generalized Quantifiers and natural language", Linguistics and Philosophy, Vol. 4.

[2] Bealer, George [1982], Quality and Concept, Oxford University Press.

[3] Bencivenga, Ernanno [1986], "Free Logics", in: D. Gabbay, and F. Guenthner, Handbook of Philosophical Logic, Dordrecht: D. Reidel Publishing Company, Vol. 3.

[4] Chierchia, Gennaro and McConnell-Ginet, Sally [1990], Meaning and Grammar. An Introduction to Semantics, MIT Press, Cambridge, Massachusetts, London, England.

[5] Cocchiarella, Nino [1986], "Frege, Russell and Logicism: a Logical Reconstruction" in: Frege Synthesized, L. Haarparanta and J. Hintikka (eds.), Dordrecht: Reidel.

[6] Dowty, R.D., Wall, R.E., and Peters, S. [1981], Introduction to Montague Semantics, Dordrecht, Boston, London: Reidel.

[7] Ebbinghaus, H. D., Flum, J., Thomas, W. Mathematical Logic [second edition 1994], Springer Verlag.

[8] Jacquette, Dale [1996], Meinongian Logic: The Semantics of Existence and Nonexistence, Berlin, New York: Walter de Gruyter \& Co.

[9] Menzel, Christopher [1986], "A complete, type-free, second order logic and its philosophical foundations", technical report \#CSLI-86-40, CSLI, Stanford University.

[10] Montague, Richard [1974], "Pragmatics", in: R. Thomason, (ed.), Formal Philosophy: Selected Papers of Richard Montague, Yale University Press, New Have \& London.

[11] Mostowski, Andrzej [1957], "On generalization of quantifiers", Fundamenta Mathematice, Vol. 44.

[12] Parsons, Terence [1998], Nonexistent Objects, Yale University Press, New Haven \& London.

[13] Paśniczek, Jacek [1998], The Logic of Intensional Objects. A Meinongian version of Classical Logic, Kluwer Academic Publishers, Dordrecht/Boston/London.

[14] Quine, Williard. V. [1963], Set Theory and Its Logic, Harvard University Press.

[15] Routley, Richard [1980], Exploring Meinong's Jungle and Beyond, Department Monograph \#3, University Canberra. 
[16] Sher, Gila [1991], The Bounds of Logic. A Generalized Viewpoint, MIT Press, Cambridge, Massachusetts, London.

[17] Westerstähl, Dag [1989], "Quantifiers in Formal and Natural languages", in Gabbay and Guenthner, Vol. IV, [1989].

[18] Zalta, Edward [1983], Abstracts Objects: An Introduction to Axiomatic Metaphysics, D. Reidel, Dordrecht.

[19] Zalta, Edward [1988], Intensional Logic and the Metaphysics of Intesionality, MIT Press, Cambridge, Massachusetts, London.

JACEK PAŚNICZEK

Maria Curie Skłodowska University

Lublin, Poland

jpasnicz@bacon.umcs.lublin.pl 